\title{
An investigation of quantum transport by the free-electron network model: Resonance and interference effects
}

\author{
Liang-Yan Hsu, Bih-Yaw Jin * \\ Department of Chemistry and Center of Theoretical Sciences, National Taiwan University, Taipei 10617, Taiwan
}

\section{A R T I C L E I N F O}

\section{Article history:}

Received 20 October 2008

Accepted 17 December 2008

Available online 25 December 2008

\section{Keywords:}

Free-electron network model

Single molecular junction

Non-equilibrium Green function

\begin{abstract}
A B S T R A C T
Quantum coherent transport through a multiply-connected network is investigated by the free-electron network model (FENM). Within this model, we study $\pi$-conjugated molecules such as benzenedithiol (BDT) in order to understand the influence of nontrivial topological structures on the transport behavior. The analytical solutions for transmission functions and $I-V$ characteristics of the simplest networked conjugated molecules are derived. Moreover, quantum effects such as resonance and interference are clearly revealed in this approach. We have also compared our FENM approach with the non-equilibrium Green's function (NEGF) method within tight-binding calculation.
\end{abstract}

() 2008 Elsevier B.V. All rights reserved.

\section{Introduction}

In 1974, Aviram and Ratner put forward the first theoretical proposal of a single molecular rectifier [1], but it received little attention owing to the limit of technology at that time. With the advances of self-assembling techniques and scanning tunneling microscopes in last two decades, their original ideas led to the birth of molecular electronics which has given rise to extensive experimental [2-10] and theoretical [11-17] investigation, thanks to its potential applications to nanoelectronic devices $[18,19]$. Additionally, single molecular electronics is also the best material to study the many-body effects in quantum transport such as Coulomb blockade [20,21], Kondo effects [20-22] and currentvoltage hysteresis [23]. For chemists, it is significant to understand the structure-conductivity relationship like chain length dependence [24], dihedral angle [10] and functional group effects [25].

Besides the effects mentioned above, the network structure also plays an important role in quantum transport [26,27]. To clarify the relation between the network structure and the electron transport property of a single molecular junction, here, in this paper, we developed a simple quantum transport theory for $\pi$-conjugated systems based on the free-electron network model (FENM) instead of using the formalisms like the Lippmann-Schwinger scattering theory [13] and the non-equilibrium Green's function (NEGF) formulation $[14,11,12]$. It is feasible to adopt the FENM to describe the most advanced nanoelectronic devices since the FENM has al-

\footnotetext{
* Corresponding author.

E-mail address: byjin@ntu.edu.tw (B.-Y. Jin).
}

ready been successfully applied to elaborate absorption spectra of conjugated systems $[28,29]$. Through the FENM, the subtle quantum effects such as resonance and interference on quantum transport can be easily revealed.

\section{Model}

In coherent quantum transport, the currents can be calculated by the well-known Landauer's formula

$I=\frac{2 e}{h} \int_{-\infty}^{+\infty} d E T(E)\left[f\left(E-\mu_{1}\right)-f\left(E-\mu_{2}\right)\right]$

which is widely used in mesoscopic physics in the past few decades. Here $\mu_{1}$ and $\mu_{2}$ are electrochemical potentials of two electrodes, $f(E)$ and $E$ stand for the Fermi function and the energy of an incident electron. $T(E)$ is the transmission function related to the molecular wires, electrodes and their couplings.

To obtain the transmission function, first of all, we adopt a direct viewpoint, a single electron scattered by effective potential, and divide the total system into three parts: a single molecular wire, junction potential, and electrodes as shown in Fig. 1. The specification of the junction and electrodes will be given in Section 4. Secondly, the molecule is regarded as a one-dimensional network, simply- or multiply-connected in two- or three-dimensional space. For instance, 1,3-butadiene can be modeled as a line, while benzene as a system with two lines connected at both ends as shown in Fig. 2. The value of effective potential is assumed to be the ionization energy of carbon atom.

The critical issue is how to calculate the transmission function of non-linear conjugated molecules like benzene. The original 


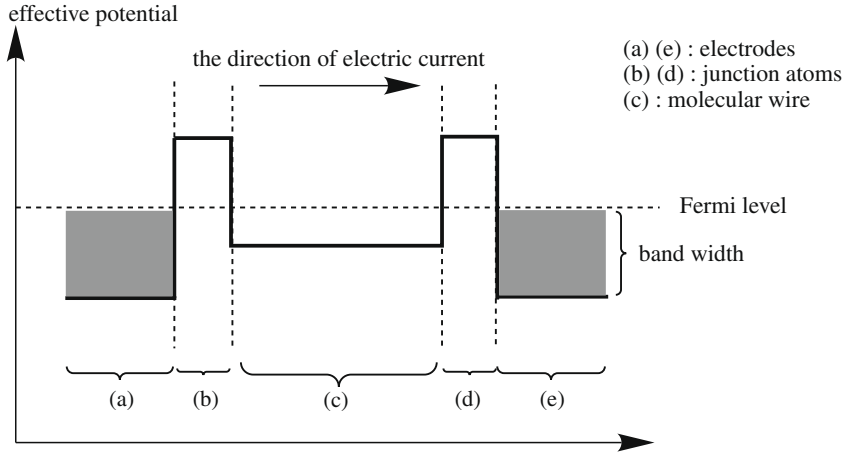

Fig. 1. Physical picture in the free-electron network model: a single electron scattered by effective potentials. For simplicity, these effective potentials are treated as stepwise potentials to capture the essential physical picture more easily.

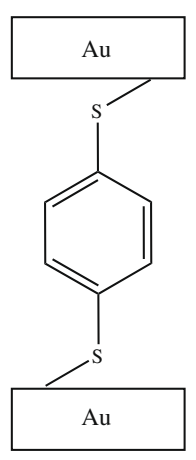

a

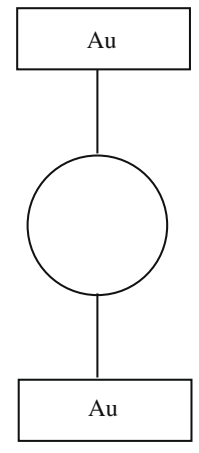

b

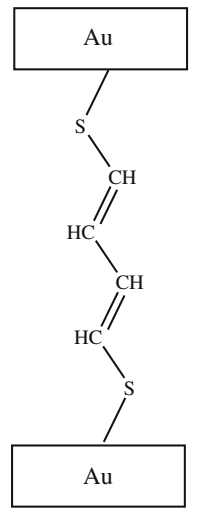

C

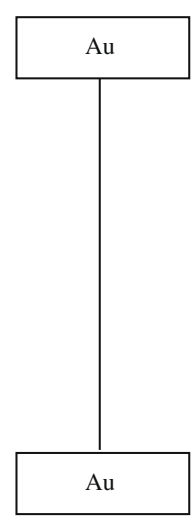

d
Fig. 2. The representation of the network model.

FENM model $[30,31]$ provides several rules to discuss the bound states of free electrons and these rules can be generalized to treat the scattering states, summarized as following:

(1) The Schrödinger equation on the branches,

$H \phi(x)=\left\{-\frac{h}{2 m} \frac{d^{2}}{d x^{2}}+V(x)\right\} \phi(x)=E \phi(x)$.

(2) The joint conditions,

(a) continuity condition

$\left[\phi_{1}\left(x_{1}\right)=\phi_{2}\left(x_{2}\right)=\phi_{3}\left(x_{3}\right)\right]_{\text {at joint }}$,

(b) conservation condition

$\sum_{B}\left(\frac{\partial \phi_{B}\left(x_{B}\right)}{\partial x_{B}}\right)_{\text {at joint }}=0$.

(3) The boundary condition,

(a) incoming wave

$\phi(x)_{\text {in }}=A e^{i k x}+B e^{-i k x}$,

(b) outgoing wave

$\phi(x)_{\text {out }}=C e^{i k x}$,

where $k=\sqrt{2 m(E-V)} / h$ and $A, B$ and $C$ are the amplitudes of incident, reflection and transmitted waves, respectively.
The relationship between current density and wavefunction is

$J=\frac{h q}{2 m i}\left(\phi^{*} \nabla \phi-\phi \nabla \phi^{*}\right)$

where $q$ is the charge quantity of an electron. Combining the Eq. (3) and the definition of transmission function, we can obtain the transmission function,

$T=\frac{J_{\text {outgoing }}}{J_{\text {incoming }}}=\frac{k_{2}}{k_{1}} \frac{|C|^{2}}{|A|^{2}}$,

where $J_{\text {incoming }}$ and $J_{\text {outgoing }}$ stand for the current densities of incident and transmitted waves, respectively. Eqs. (2)-(4) can then be employed to calculate transmission functions in this article.

\section{Transmission functions of simplest networks}

To examine the simplest network systems, we ignore junction potentials and electrodes, and consider only the situation that a single electron passes through the benzene (see Fig. 3). Fig. 3a-c depicts three different pathways and stand for para-, meta- and ortho-connections. The symbol $d$ in Fig. 3 means the $\mathrm{C}-\mathrm{C}$ bond length of benzene, so the perimeter length of benzene is $6 d$. Firstly, we discuss the case of para-connection. According to Eqs. (2)-(4), the analytical solution of transmission function is

$T=\frac{32}{41-9 \cos (k \cdot 6 d)}$.

Obviously, when $k=n \pi / 3 d$, the transmission reaches its maximum, 1 (see Fig. 4, solid line). This implies that the wavelength, $\lambda$, must satisfy the following condition:

$\lambda=\frac{2 \pi}{k}=\frac{6 d}{n}, \quad n=1,2,3, \ldots$,

i.e. the perimeter length is the integer multiples of the wavelength of its electron. Physically speaking, when this condition is satisfied, the wave is on resonance in the benzene molecule. On the other hand, if $k=(2 n+1) \pi / 6 d$, the transmission coefficient reaches its minimum value, 0.64 , because the electron wave interferes itself and forms the partial destructive interference.

The FENM can not only give the resonant wavelength, but also directly provide the information about the shapes of wavefunctions. Fig. 5A and $\mathrm{B}$ depicts the real and imaginary parts of wavefunction with $k=\pi / 3 d$ in the benzene, respectively. These<smiles>Cc1ccc(C)cc1</smiles><smiles>Cc1cccc(C)c1</smiles><smiles>Cc1ccccc1C</smiles>

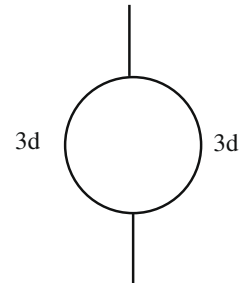

a

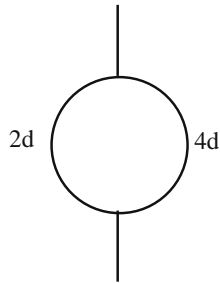

b

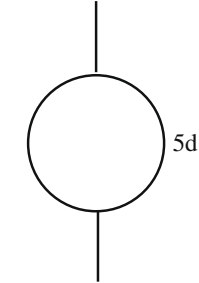

C
Fig. 3. The para-, meta-, and ortho-connection of the benzene. 


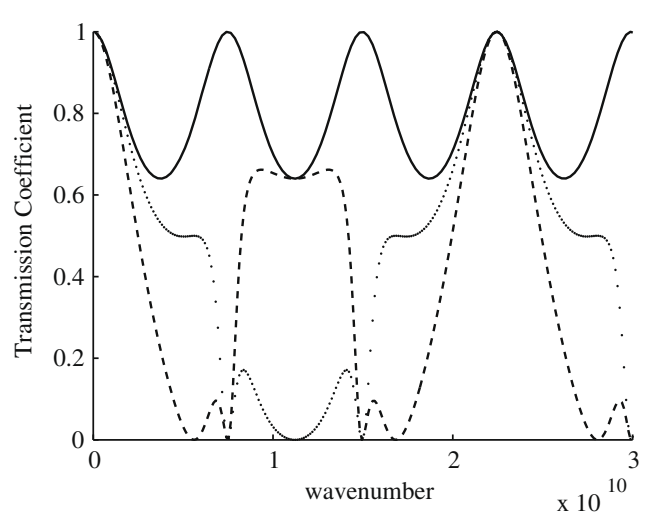

Fig. 4. The $k-T$ characteristics of benzene. The black line: the para-connection case; the dotted line: the meta-connection case; the dashed line: the ortho-connection case.
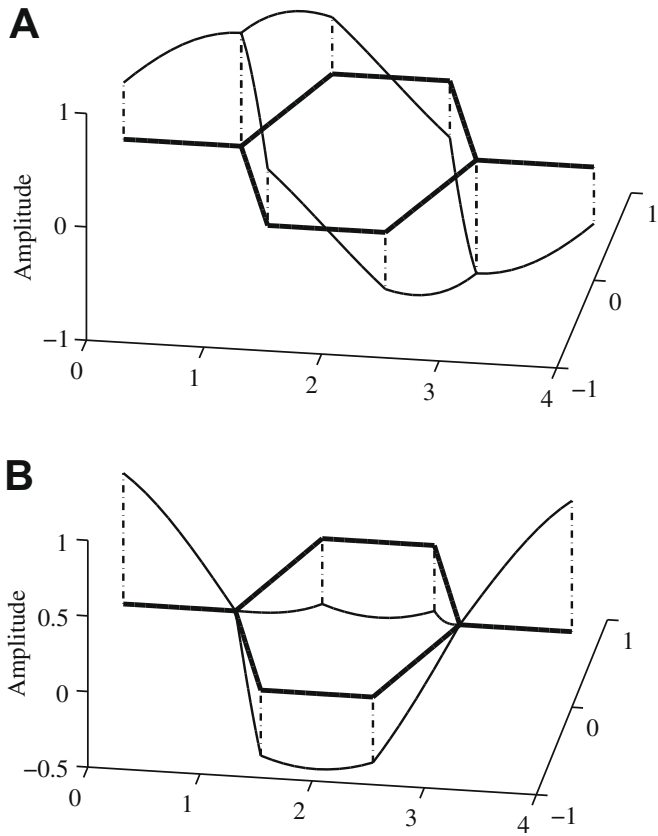

Fig. 5. The resonant states in the para-connection: $(A)$ the real part of the wavefunction; (B) the imaginary part of wavefunction. Solid line: the amplitude of the wavefunction when $k=\pi / 3 d$. Bold line: the networks of benzene. The unit of $\mathrm{x}$ and $\mathrm{y}$ axes is arbitrary.

figures show that the condition of the wavelengths and phases of resonant waves for constructive and destructive interferences in the single electron quantum transport. As shown in Fig. 5A, the incoming wave on the left-hand side consists of the incident and the reflection part, while the outgoing wave on the right-hand side contains only the transmitted part. In the middle multiple-connected region, these two waves along upper and lower pathways have the same real part and form completely constructive interference with $T=1$. The same conclusion can be drawn from Fig. 5B since the two waves in the middle have the same imaginary part.

In the meta-connection case, the transmission function is given by the following lengthy expression:

$T=32 \cos ^{2}(k d)[1+2 \cos (2 k d)]^{2} / X$,

where the denominator, $X$, is given by

$$
\begin{aligned}
X= & 114+154 \cos (2 k d)+64 \cos (4 k d)-17 \cos (6 k d) \\
& -18 \cos (8 k d)-9 \cos (10 k d) .
\end{aligned}
$$

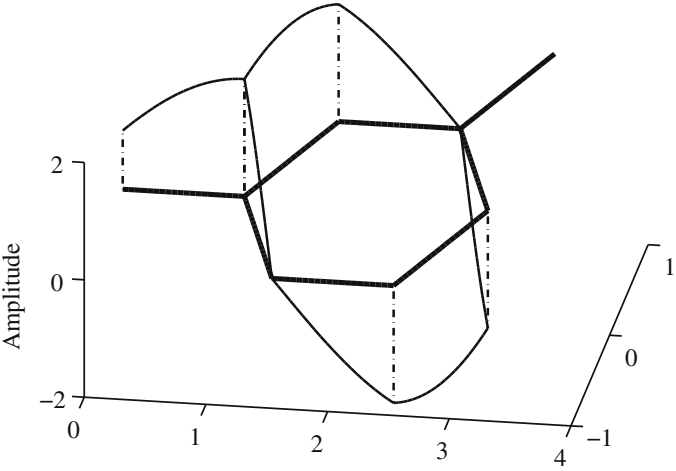

Fig. 6. The real part of the wavefunction for the anti-resonance state with $k=\pi / 3 d$ in the meta-connection case. This wave forms destructive interference.

The transmission function for this case is depicted as the dotted line in Fig. 4. We find the transmission reaches its maximum value 1 when $k=n \pi / d$ as required by the analytical expression of transmission function given by Eq. (7). In this case the completely constructive interference occurs for resonant waves with the wavelength, $2 d / n$. On the other hand, if wavevectors satisfy the conditions, $k=(3 n+1) \pi / 3 d,(3 n+2) \pi / 3 d$ and $k=(2 n+1) \pi / 2 d$, the transmission falls to zero. In this situation, the wave forms an anti-resonance state, leading to the completely destructive interference in the benzene. The phase of the anti-resonance state is sketched in Fig. 6. Obviously, the real parts of two waves just before entering the outgoing channel have opposite signs, making the amplitude of outgoing wave equal to zero. Besides, note that the conditions, $k=\pi / 3 d$ and $2 \pi / 3 d$, also correspond to the standing waves of a free particle in a ring. This shows that it is not adequate to determine the properties of electron transport only by the MOs of isolated molecules. Comparing the para- and meta-connection cases, the different networks have indeed a great influence on the transmission function of systems [33].

The transmission function of the ortho-connection is also shown in Fig. 4 (the dashed line). However, we do not intend to show the analytical form of the transmission function of the benzene with the ortho-connection since it is too complicated. The para-connection case has given us a clear physical picture that an incoming electron may interfere with itself in the molecular wire very similar to the double-slit experiment. The wavenumbers and wavelengths of resonant states and anti-resonant states in the different network of the benzene are summarized in Tables 1 and 2 .

Table 1

The wavenumber $(k)$ of resonant states and anti-resonant states of the benzene with

\begin{tabular}{|c|c|c|c|}
\hline Type of connections & Para-connection & Meta-connection & Ortho-connection \\
\hline $\begin{array}{l}\text { Resonant states } \\
\quad(T=1)\end{array}$ & $k=n \pi / 3 d$ & $k=n \pi / d$ & $k=n \pi / d$ \\
\hline $\begin{array}{l}\text { Anti-resonant } \\
\text { states }(T=0)\end{array}$ & None & $\begin{array}{l}k=(3 n+1) \pi / 3 d \\
k=(3 n+2) \pi / 3 d \\
k=(2 n+1) \pi / 2 d\end{array}$ & $\begin{array}{l}k=(3 n+1) \pi / 3 d \\
k=(3 n+2) \pi / 3 d\end{array}$ \\
\hline
\end{tabular}
the para-, the meta-, and the ortho-connection.

Table 2

The wavelength $(\lambda)$ of resonant states and anti-resonant states of the benzene with the para-, the meta-, and the ortho-connection.

\begin{tabular}{llll}
\hline Type of connections & Para-connection & Meta-connection & Ortho-connection \\
\hline Resonant states & $\lambda=6 d / n$ & $\lambda=2 d / n$ & $\lambda=2 d / n$ \\
$\quad(T=1)$ & \multirow{2}{*}{ None } & $\lambda=6 d /(3 n+1)$ & $\lambda=6 d /(3 n+1)$ \\
Anti-resonant & & $\lambda=6 d /(3 n+2)$ & $\lambda=6 d /(3 n+2)$ \\
$\quad$ states $(T=0)$ & & $\lambda=4 d /(2 n+1)$ & \\
& &
\end{tabular}



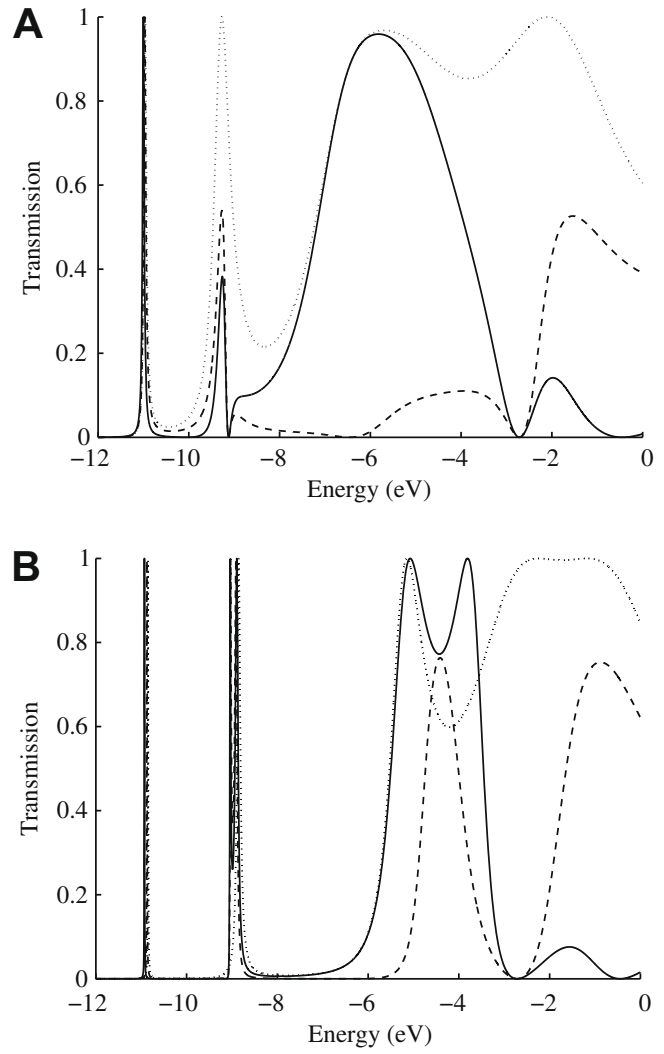

Fig. 7. Transmission functions of benzenedithiol with the para-, the meta-, and the ortho-connection in zero electric field: (A) Strong coupling. (B) Weak coupling. The solid line is the ortho-connection. The dashed line is the meta-connection. The dotted line is the para-connection.

\section{Transmission functions for phenyl dithiol junction}

In addition to the simple model systems considered in the previous section, here we show that the FENM can also be employed to model the more realistic nanoelectric devices. In this section, we will discuss the transmission functions of phenyl dithiol in the low bias limit with either strong or weak coupling to the electrodes.

To model Reed's experiment, the electrodes and junction atoms must be considered. As usual, the electrodes are modeled as a noninteracting electron gas. The Fermi level is taken as $-10.4 \mathrm{eV}$ [12]; the value of band edge is $-15.93 \mathrm{eV}$ based on the argument of the non-interacting electron gas model [32]. ${ }^{1}$ In the part of junction atoms, the diameter of a sulfur atom is assumed as $2.13 \AA$ based on the Reed's experiments [2]. The effective potential on the sulfur atom is regarded as a tunable parameter. In the strong-coupling limit between the molecular wire and electrodes, the effective potential is chosen to be $-10 \mathrm{eV}$; while in the weak-coupling limit, it is set to be $-7 \mathrm{eV}$.

Fig. 7 shows that the transmission functions of phenyl dithiol junction in the strong and weak coupling limits. In the strong-coupling situation (Fig. 7A), the transmissions for the situations with meta- and ortho-connections fall down to zero at $-2.7 \mathrm{eV}$ and $-9.1 \mathrm{eV}$, corresponding to the resonant waves with wavelengths $4.2 \AA$ and $8.4 \AA$, respectively. These two wavelengths are consistent with the condition of anti-resonant states in Table $2(d=1.4 \AA$, $n=0$ and $\lambda=6 d /(3 n+1)$ or $\lambda=6 d /(3 n+2))$. Additionally, in the meta-connection case, the transmission also vanishes at $-6.5 \mathrm{eV}$, in agreement with the condition of anti-resonant states in Table 2

\footnotetext{
${ }^{1}$ For gold electrode, the kinetic energy is $5.53 \mathrm{eV}$.
}

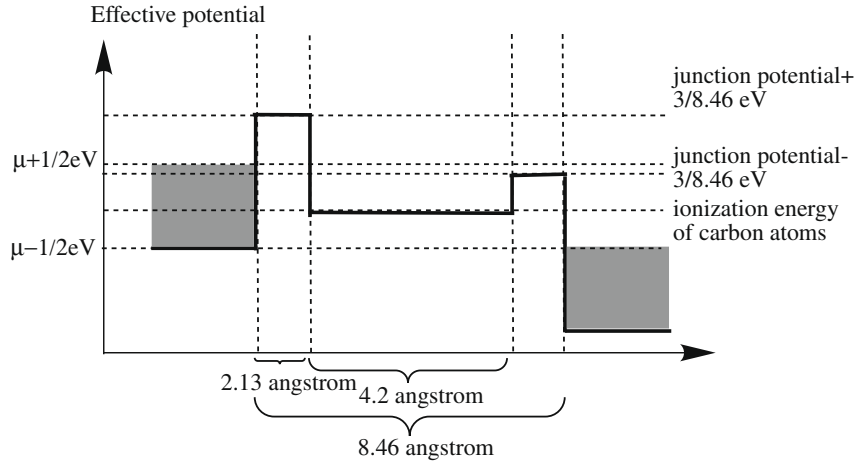

Fig. 8. An electron is scattered by effective potentials under non-zero electric field $\mu_{L}+1 / 2 \mathrm{eV}$ and $\mu_{R}-1 / 2 \mathrm{eV}$ stand for the effective potential of the left electrode and the right electrode, $U_{\text {junction }}+3 / 8.46 \mathrm{eV}$ is the left junction potential, $U_{\text {junction }}-3 / 8.46 \mathrm{eV}$ is the right junction potential, and the effective potential of the molecular wire remains the same.

$(d=1.4 \AA, n=0$ and $\lambda=4 d /(2 n+1))$. The anti-resonances in the transmission functions arise from the interference among different coherent transport pathways through a multiply-connected network, in agreement with other works using different approaches [34-36]. They will not disappear by changing different types of junctions between molecular wire and electrodes.

Besides, in the strong coupling case (Fig. 7A), the widths of the resonances are generally broader than those in the weak coupling case (Fig. 7B). It makes sense since the height of the effective potential at junction atoms represents the interaction between the molecular wire and electrodes. When the coupling is weaker, the position of resonant states is closer to the MOs of isolated molecular wires.

Incidentally, the effect of non-zero electric field at the finite bias can also be considered by using the symmetric [11,12] and stepwise effective potential as shown in Fig. 8. However, since the transmission functions under electric field do not show significant difference from those in the zero-bias limit, the results are not shown here.

\section{I-V characteristics for benzenedithiol junction}

In this section, we will discuss the $I-V$ and $G-V$ characteristics based on the FENM model, and then compare the results with the transmission functions calculated by the NEGF method at the level of the tight-binding approximation (NEGF-TB).

In the strong coupling limit, the $I-V$ and $G-V$ characteristics of BDT with the para-connection are shown in Fig. 9. The solid line reveals non-linear relationship between bias and current. The first

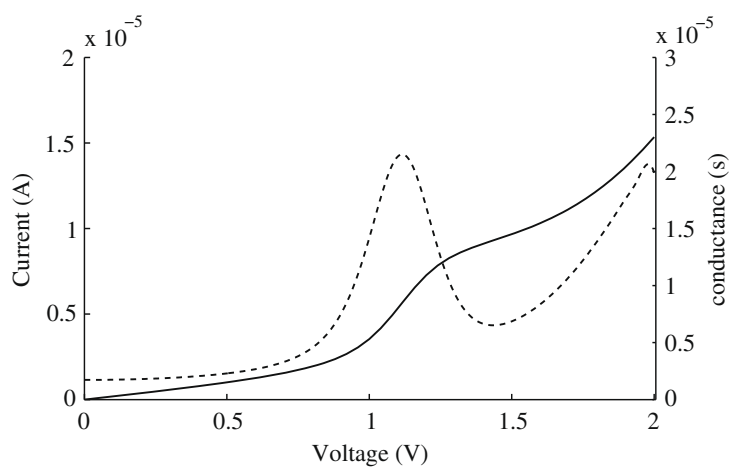

Fig. 9. The $I-V$ characteristics and $G-V$ characteristics of benzenedithiol calculated by the FENM model. The solid line: $I-V$ characteristics. The dashed line: $G-V$ characteristics. 
and the second peaks of $G-V$ characteristics (dashed line) correspond to the first and the second resonant states, respectively. The conductance start to rise at about $0.7 \mathrm{eV}$, in agreement with Reed's experiments in the low bias region. In the medium and high bias regions $(>2.0 \mathrm{eV})$, owing to the neglect of other interactions such as electron-electron, electron-phonon, and electron-photon couplings, the calculated $I-V$ characteristics dose not match the experimental result perfectly, hence we do not attempt to explain $I-V$ and $G-V$ characteristics of medium and high bias regions. Nevertheless, this simple model seems to capture main physical pictures in the low bias limit.

At the end of the section, we will compare the transmission functions calculated by the FENM with by the NEGF-TB approach [37-39]. In the NEGF, the transmission function is [40]: $T(E)=$ $\operatorname{Tr}\left[\Gamma_{L} G^{R} \Gamma_{R} G^{A}\right]$, where $\Gamma_{L(R)}$ is the spectral function of left (right) electrode. $G^{R(A)}$ is the retarded (advanced) molecular Green's function. The spectral function and the retarded molecular Green's function could be represented as

$$
\begin{aligned}
& \Gamma=i\left[\Sigma^{R}-\Sigma^{A}\right]=-2 \operatorname{Im}\left[\Sigma^{R}\right], \\
& G^{R}=\frac{1}{E-H_{\mathrm{mol}}-\Sigma^{R}},
\end{aligned}
$$

where $H_{\text {mol }}$ is molecular Hamiltonian. To simplify calculation, the tight-binding Hamiltonian is adopted. $\Sigma^{R}$ is the retarded self-energy treated with the Newns-Anderson model defined by

$$
\begin{aligned}
& \Sigma(E)=\Lambda(E)-i \Delta(E), \\
& \Lambda(E)= \begin{cases}\frac{V_{K}^{2}}{\gamma} E / 2 \gamma, & |E / 2 \gamma|<1, \\
\frac{V_{K}^{2}}{\gamma}\left\{E / 2 \gamma+\left[(E / 2 \gamma)^{2}-1\right]^{1 / 2}\right\}, & E / 2 \gamma<-1, \\
\frac{V_{K}^{2}}{\gamma}\left\{E / 2 \gamma-\left[(E / 2 \gamma)^{2}-1\right]^{1 / 2}\right\}, & E / 2 \gamma>1,\end{cases} \\
& \Delta(E)= \begin{cases}\frac{V_{K}^{2}}{\gamma} \sqrt{1-(E / 2 \gamma)^{2}}, & |E / 2 \gamma|<1, \\
0, & \text { otherwise. }\end{cases}
\end{aligned}
$$

In this model, we use $V_{k}$ to characterize the coupling between electrodes and the molecular wire. The index $k$ stands for the $k$ th site of molecular wire. We assume $V_{1}=V_{N}=V$ and the other couplings are set to be zero, i.e. the couplings only exist at the end sites of molecular wires and electrodes. $E$ is the energy of the electron measured from the center of energy band in the electrode. $2 \gamma$ is the bandwidth of electrodes.

Based on these approximations, we can find the transmission function as the following:

$$
T(E)=\left(\frac{2 V^{2}}{\gamma} \sqrt{1-(E / 2 \gamma)^{2}}\right)^{2}\left|G_{1 N}^{R}\right|^{2} .
$$

The transmission functions of benzene with the para-, the meta-, the ortho-connections are shown in Fig. 10. Comparison with Fig. 7A leads to the following discussions: First, in the para-connection case, the resonant states could have good one-to-one correlation between the FENM and the NEGF-TB approach: $-11.0 \mathrm{eV}$ to $-12 \mathrm{eV},-9.3 \mathrm{eV}$ to $-10 \mathrm{eV}$, and $-5.7 \mathrm{eV}$ to $-6 \mathrm{eV}$. These three resonant states are also the eigenstates of benzene. Second, in the meta- and the ortho-connection cases, the transmission functions reach a zero at about $-10 \mathrm{eV}$ and $-6 \mathrm{eV}$. These two states are anti-resonant states and correspond to the positions at $-9.1 \mathrm{eV}$ and $-2.7 \mathrm{eV}$ from the FENM. Third, in the meta-connection case, the transmission function diminishes to a zero at $-8 \mathrm{eV}$. The same result could be found at $-6.5 \mathrm{eV}$ in the FENM. Obviously, the transmission functions calculated by the FENM are in good agreement with those by the NEGF-TB.

This agreement seems to be not a coincidence, as has been demonstrated during the fifties by Chicagos group, there is a close

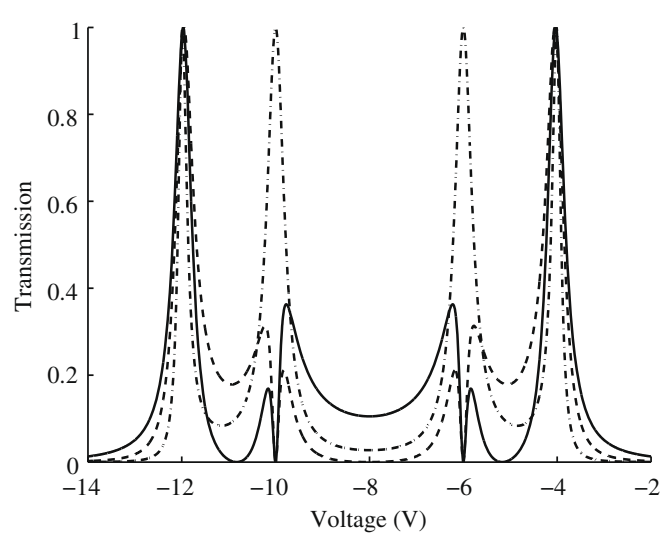

Fig. 10. Transmission functions of benzene in zero electric field calculated by the NEGF-TB model. The solid line: the ortho-connection case. The dashed line: the meta-connection case. The dotted line: the para-connection case. Parameters of tight-binding model: $\alpha=-8 \mathrm{eV}, \beta=-2 \mathrm{eV}$. Parameters of the Newns-Anderson model: $V=1 \mathrm{eV}, \gamma=3 \mathrm{eV}$.

correspondence between the FENM and LCAO MO approaches [29]. Moreover, a formal equivalence can be established in the case that LCAO MO's approach contains only one orbital per atom [31]. When FENM is applied to quantum transport through a single-molecule junction, the most important difference between the FENM and NEGF-TBM approaches is the neglect of the self-energy due to the coupling to the electrodes.

The FENM approach provides a clear physical picture to help us understand the resonance and interference effects in quantum transport. Additionally, it also can capture the main characteristics of the advanced nanoelectric devices. However, there are still plenty of rooms for the improvement of this model. In particular, it seems worthy to develop a general formulation based on the FENM to the more general multiply-connected networks such as polyaromatic hydrocarbons, fullerene compounds and other molecular circuits.

\section{Acknowledgements}

We wish to thank the National Science Council, Taiwan for the financial support. Hsu wishes to thank Mr. Fan for useful discussions.

\section{References}

[1] A. Aviram, M.A. Ratner, Chem. Phys. Lett. 29 (1974) 277.

[2] M.A. Read, C. Zhou, C.J. Muller, T.P. Burgin, J.M. Tour, Science 278 (1997) 252.

[3] J. Chen, M.A. Reed, A.M. Rawlett, J.M. Tour, Science 286 (1999) 1550.

[4] J. Chen, W. Wang, M.A. Reed, A.M. Rawlett, D.M. Price, J.M. Tour, Appl. Phys. Lett. 77 (2000) 1224.

[5] D. Porath, A. Bezryadin, S. Vries, C. Dekker, Nature 403 (2000) 635.

[6] X.D. Cui, A. Primak, X. Zarate, O.F. Sankey, A.L. Moore, T.A. Moore, D. Gust, G. Harris, S.M. Lindsay, Science 294 (2001) 571.

[7] R.H.M. Smit, Y. Noat, C. Untiedt, N.D. Lang, M.C. van Hemert, J.M. van Ruitenbeek, Nature 419 (2002) 906.

[8] B. Xu, N.J. Tao, Science 301 (2003) 1221.

[9] T. Dadosh, Y. Gordin, R. Krahne, I. Khivrich, D. Mahalu, V. Frydman, J. Sperling, A. Yacoby, I. Bar-Joseph, Nature 436 (2005) 677.

[10] L. Venkataraman, J.E. Klare, C. Nuckolls, M.S. Hybersten, M.L. Steigerwald, Nature 442 (2006) 904.

[11] S. Datta, W. Tuan, H. Song, R. Reifenberger, J.I. Henderson, C.P. Kubiak, Phys. Rev. Lett. 79 (1997) 2530.

[12] S. Datta, W. Tuan, H. Song, R. Reifenberger, J.I. Henderson, C.P. Kubiak, J. Chem. Phys. 109 (1998) 2874.

[13] M. Di Ventra, S.T. Pantelides, N.D. Lang, Phys. Rev. Lett. 84 (2000) 979

[14] Y. Xue, S. Datta, M.A. Ratner, Chem. Phys. 281 (2002) 151

[15] R. Gebauer, R. Car, Phys. Rev. B 70 (2004) 125324.

[16] K. Burke, R. Gebauer, R. Car, Phys. Rev. Lett. 94 (2005) 146803.

[17] T. Shimazaki, Y. Xue, M.A. Ratner, K. Yamashita, J. Chem. Phys. 124 (2006) 114708.

[18] C. Joachim, J.K. Gimzewski, A. Aviram, Nature 408 (2000) 541 
[19] N.J. Tao, Nat. Nanotechnol. 1 (2006) 173.

[20] J. Park, A.N. Pasupathy, J.I. Goldsmith, C. Chang, Y. Yaish, J.R. Petta, M. Rinkoski, J.P. Sethna, H.D. Abruna, P.L. McEuen, D.C. Ralph, Nature 408 (2000) 722.

[21] W. Liang, M.P. Shores, M. Bockrath, J.R. Long, H. Park, Nature 408 (2000) 725.

[22] W.J. Liang, M.P. Shores, M. Bockrath, J.R. Long, H. Park, Nature 417 (2002) 6890

[23] T.L. Schull, J.G. Kushmerick, C.H. Patterson, C. George, M.H. Moore, S.K. Pollack, R. Shashidhar, J. Amer. Chem. Soc. 125 (2002) 3202

[24] R.E. Holmlin, R. Haag, M.L. Chabinyc, R.F. Ismagilov, A.E. Cohen, A. Terfort, M.A. Rampi, G.M. Whitesides, J. Amer. Chem. Soc. 123 (2001) 5075.

[25] L. Venkataraman, Y.S. Park, A.C. Whalley, C. Nuckolls, M.S. Hybertsen, M.L. Steigerwald, Nano Lett. 7 (2007) 501.

[26] G.C. Solomon, D.Q. Andrews, T. Hansen, R.H. Goldsmith, M.R. Wasielewski, R.P. Van Duyne, M.A. Ratner, J. Chem. Phys. 129 (2008) 054701.

[27] L.Y. Hsu, Q.R. Huang, B.Y. Jin, J. Phys. Chem. C 112 (2008) 10538.

[28] H. Kuhn, J. Chem. Phys. 16 (1948) 840.
[29] J.R. Platt, K. Ruedenberg, C.W. Scherr, N.S. Ham, H. Labhart, W. Lichten, FreeElectron Theory of Conjugated Molecules: A Source Book, Papers of the Chicago Group 1949-1961, John-Wiley \& Sons, Inc., 1964.

[30] K. Ruedenberg, J. Chem. Phys. 21 (1953) 1565.

[31] K. Ruedenberg, J. Chem. Phys. 22 (1954) 1878.

[32] C. Kittel, Introduction to Solid State Physics, Wiley, 1996

[33] L.Y. Hsu, B.Y. Jin, Chem. Phys. Lett. 457 (2008) 279.

[34] P.A. Orellana, F. Dominguez-Adame, I. Gomez, M.L. Ladron de Guevara, Phys. Rev. B 67 (2003) 085321.

[35] D. Walter, D. Neuhauser, R. Baer, Chem. Phys. 299 (2004) 139.

[36] D. Brisker, I. Cherkes, C. Gnodtke, D. Jarukanont, S. Klaiman, W. Koch, S. Weissman, R. Volkovich, M.C. Toroker, U. Peskin, Mol. Phys. 106 (2008) 281.

[37] V. Mujica, M. Kemp, M.A. Ratner, J. Chem. Phys. 101 (1994) 6849.

[38] V. Mujica, M. Kemp, M.A. Ratner, J. Chem. Phys. 101 (1994) 6856.

39] S. Datta, Quantum Transport: Atom to Transistor, Cambridge, 2005.

[40] D.S. Fisher, P.A. Lee, Phys. Rev. 23 (1981) 6851. 